\title{
Resultados del primer registro nacional de fibrosis pulmonar idiopática en Chile
}

\author{
ALVARO UNDURRAGA P. ${ }^{1}$, MATÍAS FLORENZANO V. ${ }^{1}$, LUISA MONTECINO L. ${ }^{1}$ (QEPD), \\ J. CARLOS RODRÍGUEZ D. ${ }^{1}$, MAURICIO SALINAS F. ${ }^{1}$, MARÍA ANGÉLICA BELLO F. ${ }^{2}$, \\ ISABEL DE LA FUENTE M. ${ }^{3}$, GINO FUENTES L. ${ }^{4}$, CLAUDIO GALLARDO N. ${ }^{5}$, \\ MÓNICA GUTIÉRREZ C. ${ }^{6}$, JUAN C. MAURELIA G. ${ }^{7}$, MÓNICA MENESES M. ${ }^{4}$, \\ MIRTA REYES H. ${ }^{8}$, PATRICIO RIOSECO S. ${ }^{9}$, ROSA ELENA ROLDÁN T. ${ }^{10}$, \\ LUIS A. SOTO G. ${ }^{11}$, TULIO SOTO T. ${ }^{12}$, RICARDO TOBAR H. (QEPD) ${ }^{13}$, \\ EDUARDO VERGARA LL. ${ }^{14}$, GABRIEL CAVADA CH. ${ }^{15}$ y participantes del grupo EPD Chile*
}

\section{Results of the first national register of idiopathic pulmonary fibrosis in Chile}

Introduction: Idiopathic pulmonary fibrosis is the most common idiopathic interstitial pneumonia, with incidence and prevalence figures varying worldwide because of the inconsistent way of collecting cases in different studies. There are no published figures on the epidemiology of IPF in Chile or Latin America. It is relevant to know them because of the health burden of patients with IPF and the recent approval for treatment purposes of high cost antifibrotic drugs. The objective of this study was to develop a clinical registry of patients with IPF treated by Chilean pulmonologists from different regions of the country, using the diagnostic means they usually use in real life. Patients and Methods: An online electronic survey was designed by the group of diffuse pulmonary diseases of the "Instituto Nacional del Tórax" to register patients with diagnosis of IPF from June 2015 to June 2017 according to ATS/ERS/JRS/ALAT criteria. Results: 40 of the 200 invited pulmonologists sent IPF cases from the country's 13 regions, completing a total of 700 patients. 2/3 were old cases, a similar number of men and women, $73 \%$ had definitive UIP pattern in CT, the majority were patients over 60 years old and in only 16\% biopsy was requested for diagnosis. Conclusions: A register of 700 cases represents a very important number of patients with IPF in Chile that allows us to approach the characterization of the cohort and to strengthen a network of specialists dedicated to the care of these patients and their families.

Key words: Idiopathic Pulmonary Fibrosis; Humans; Registries; Survey and Questionnaires; Pulmonologists, Chile.

Fuentes de financiamiento: Este estudio contó con apoyo de financiamiento del programa computacional de la Sociedad Chilena de Enfermedades Respiratorias. Además la enfermera coordinadora del proyecto recibió un estipendio mensual del Laboratorio Abbott Chile.

\footnotetext{
${ }^{1}$ Instituto Nacional del Tórax.

${ }^{2}$ Hospital San Juan de Dios.

${ }^{3}$ DIPRECA.

${ }^{4}$ Hospital Base de Valdivia.

${ }^{5}$ Hospital Barros Luco.

${ }^{6}$ Hospital Naval de Viña del Mar.

${ }^{7}$ Hospital de Copiapó.

${ }^{8}$ Hospital Carlos Van Buren.

${ }^{9}$ Hospital Las Higueras, Talcahuano.

${ }^{10}$ Hospital San José.

${ }^{11}$ Hospital de Coquimbo.

${ }^{12}$ Hospital Regional de Talca.

${ }^{13}$ Hospital San Borja Arriarán.

${ }^{14}$ Universidad de Antofagasta.

${ }^{15}$ Universidad de los Andes.

${ }^{16} \mathrm{Hospital}$ de Iquique .
} 


\section{Resumen}

Introducción: La FPI es la neumonía intersticial idiopática más común, con cifras de incidencia y prevalencia que varían en el mundo por la poco uniforme manera de recolectar casos en los diferentes estudios. No hay cifras publicadas sobre la epidemiología de la FPI en Chile ni Latinoamérica. Se hace relevante conocerlas por la carga sanitaria que representan los pacientes con FPI y por la aprobación reciente de drogas antifibróticas de alto costo. El objetivo de este estudio fue realizar un registro de pacientes con FPI atendidos por neumólogos chilenos de diversos regiones del país, con los medios diagnósticos que habitualmente utilizan en la vida real. Pacientes y Métodos: Se utilizó una encuesta electrónica en línea diseñada por el grupo de enfermedades pulmonares difusas del Instituto Nacional del Tórax para registro de pacientes con diagnóstico de FPI según criterios ATS/ERS/JRS/ALAT desde junio de 2015 a junio de 2017. Resultados: 40 de los 200 neumólogos invitados enviaron casos de FPI de las 13 regiones del país, completando un total de 700 pacientes. $2 / 3$ eran casos antiguos, un número similar de hombres y mujeres, 73\% tienen patrón definitivo de UIP (Usual Interstitial Pneumonia) en tomografía axial computarizada, la mayoría eran pacientes sobre 60 años y en solo $16 \%$ se solicitó biopsia para diagnóstico. Conclusiones: Un registro de 700 casos representa un número muy importante de pacientes con FPI en Chile que nos permite acercarnos a la caracterización de la cohorte y a fortalecer una red de especialistas dedicados al cuidado de estos pacientes y sus familias.

Palabras clave: Fibrosis pulmonar idiopática; seres humanos; registros; Encuestas y cuestionarios; Neumólogos; Chile.

\section{Introducción}

La fibrosis pulmonar idiopática (FPI) pertenece al grupo de las enfermedades pulmonares difusas. Es una enfermedad de predominio masculino, que se presenta sobre los 60 años. Se manifiesta con tos y disnea progresiva con cursos evolutivos de velocidades variables. En la radiografía de tórax se demuestra una enfermedad pulmonar difusa con volúmenes habitualmente disminuidos. La tomografía axial computada (TAC) demuestra en al menos el 50\% de los casos un patrón radiológico característico. A pesar de lo variable de la historia natural de esta condición, la sobrevida promedio de los pacientes es de 3 a 5 años sin disponerse hasta hace muy poco de alternativas terapéuticas con algún grado de eficacia. El año 2012 se publicó una experiencia dirigida por US National Institutes of Health (NIH) que demuestra que el tratamiento usado por dos décadas en todo el mundo con Prednisona, Azatriopina, y N-Acetil Cisteína no sólo no produce beneficios sino que es habitualmente deletéreo para estos pacientes ${ }^{1}$.

Muy recientemente se han incorporado dos nuevas terapias con las nuevas drogas Pirfenidona y Nintedanib que en estudios controlados, randomizados placebo-controlados, con adecuados tamaños muestrales, han demostrado su utilidad ${ }^{2-3}$.

La FPI es la Neumonía Intersticial Idiopática más común. La información de registros sugieren que representa de 17 a $37 \%$ de todas las enfermedades pulmonares difusas y aún en algunas series estaría sobre el $50 \%{ }^{4-5}$. Sin embargo, se desconocen cifras exactas de incidencia y prevalencia, ya que existe una gran dispersión en las cifras por múltiples razones ${ }^{6-7}$. En efecto, en los estudios realizados hasta el momento no se han usado definiciones uniformes lo cual se entiende porque los criterios para definiciones precisas de la enfermedad son relativamente recientes. Por ello el gold standard diagnóstico de la enfermedad es difícil de definir. En los últimos años se ha hecho claro que el cuadro clínico, y los hallazgos de la Tomografía Computarizada de alta resolución pueden ser diagnósticos en aproximadamente el $50 \%$ de los casos y en el resto se puede requerir una biopsia pulmonar. Esto ha aumentado la precisión diagnóstica lo que "acrecienta" la mortalidad por lo que la dependencia de la tecnología para el diagnóstico también es una limitante para la medición en la población general.

Para estimar la prevalencia de la enfermedad se han utilizado diversas metodologías para buscar casos como los diagnósticos de los egresos hospitalarios, los certificados de defunción o encuestas entre médicos con diferentes especialidades. Los códigos de clasificación tampoco han sido de mucha utilidad de clasificación. La última modificación de la clasificación internacional de enfermedades tampoco resuelve el problema pues en el acápite de "otras enfermedades que afectan principalmente al intersticio" (J84) propone varias afecciones que pueden dar lugar a confusiones por sobreponerse con otras patologías pulmonares ${ }^{8}$. 
Hay diversas publicaciones que se refieren incidencia y prevalencia de la enfermedad. Un estudio reciente que recolectó datos de beneficiarios mayores de 65 años pertenecientes al sistema de seguros Medicare en Estados Unidos, entre 2001 y 2011, mostró tasas con una incidencia acumulada de 93,7 casos por 100.000/año, y una prevalencia que va desde 202,2 casos por 100.000 en 2001 a 494,5 casos por 100.000 en 2011. En el período observado la incidencia permaneció estable, mientras que la prevalencia aumentó, lo que sugiere que los casos han aumentado más su sobrevida ${ }^{9}$. En el Reino Unido, un primer estudio reportó una densidad de incidencia de 4,6 por 100.000/año obteniendo casos de una base de datos longitudinal de atención primaria entre 1991 y 2003, Usando la información de la misma base de datos se reportó una densidad de incidencia de 7,4/100.000 año para el período 2000-2009 ${ }^{10-}$ ${ }^{11}$. Ello sugiere también que la incidencia de la enfermedad podría estar aumentando.

Cuando los datos se han estratificado por género y edad, la enfermedad es más común en hombres y de mayor edad, especialmente por encima de los 75 años. Un reciente estudio japonés que exploró los certificados de beneficios médicos por FPI en la prefectura de Hokkaido entre los años 2003 y 2007, reportó prevalencia y densidad de incidencia de 10,0 y 2,3 por $100.000 /$ año respectivamente ${ }^{12}$. Aunque se confirma el predominio en hombres y los de mayor edad en pacientes asiáticos, las tasas son menores que en países occidentales, lo que siguiere un posible rol de diferencias étnicas.

En Chile no tenemos ninguna cifra publicada sobre la realidad epidemiológica de esta enfermedad. Sin embargo, es experiencia de todos los neumólogos, que la enfermedad se ha transformado desde constituir una condición infrecuente en una patología relativamente frecuente que llega a la consulta de enfermedades respiratorias y de hecho en varios centros neumológicos del país se han formado policlínicos especiales para atender a estos pacientes.

\section{Objetivos del estudio}

El objetivo principal de este estudio fue conformar un registro de la Fibrosis Pulmonar Idiopática diagnosticada en la actualidad por los neumólogos chilenos de diversas regiones de nuestro país entre 2015 y 2017, con los medios diagnósticos que habitualmente usan estos especialistas en sus prácticas clínicas. Los objetivos secundarios fueron:
1. Estudiar las cifras obtenidas y evaluar el porcentaje de Neumonia Intersticial Usual (NIU) versus posibles NIU.

2. Analizar algunas características demográficas y clínicas básicas de los pacientes incluidos en la encuesta.

3. Conocer la cobertura de realización de una biopsia pulmonar como medio de diagnóstico de la condición.

\section{Pacientes y Métodos}

El grupo de enfermedades pulmonares difusas del Instituto Nacional del Tórax, con el patrocinio de la Sociedad Chilena de Enfermedades Respiratorias, organizó un registro vía página web para que los especialistas en enfermedades respiratorias del país registraran los pacientes con diagnóstico de FPI que atendieran durante un año ya sea pacientes nuevos o controles. Se trata de un estudio descriptivo, transversal, observacional en base a dicho registro.

Se diseñó una encuesta simple, de una página, que pretendió recoger en forma esquemática, las enfermedades pulmonares difusas que pudieran corresponder a FPI, a través de datos clínicos, y de la interpretación del TAC (hecho por el neumólogo con o sin colaboración de su grupo de trabajo, tal como lo realiza en su práctica diaria) durante un año. Se trataba de las FPI seguras o posibles, siguiendo las definiciones que sugiere el Consenso de las Sociedades de Tórax Americana, Europea, Japonesa y Latino Americana ${ }^{13}$. En la encuesta se incorporó una reproducción de los criterios radiológicos de NIU (UIP) y de posible NIU (UIP) de acuerdo a lo publicado por el consenso referido. El tiempo de recogida de datos comprendió el período entre junio de 2015 a junio de 2017. En la misma encuesta se adjuntaba un instructivo que aclaraba cada punto de ese instrumento.

Se invitó a participar a todos los médicos neumólogos chilenos que trabajan en hospitalización y consulta en el sistema público y privado a través de todo el país de acuerdo a registro de la Sociedad Chilena de Enfermedades Respiratorias. Se prefirió los especialistas registrados en esta Sociedad pues el diagnóstico de FPI generalmente lo realizan los neumólogos.

Cada caso registrado obtuvo un número correlativo por región del país sin incluir el nombre, por razones de confidencialidad, pero si el número de RUT (DNI cautelando la privacidad de estos antecedentes). Cada médico participante debía explicar al paciente el objetivo de la en- 
cuesta y solicitar su incorporación mediante un consentimiento informado simple que debería guardó el investigador local. Cada formulario de encuesta fue enviado por vía electrónica al nivel central donde fue registrado y analizado. A cada profesional participante se le ofreció asesoría en la interpretación y el manejo de pacientes por el grupo de EPD (enfermedades pulmonares difusas) del Instituto Nacional del Tórax, el cual sesiona semanalmente en análisis semejantes. Trimestralmente se realizó un análisis parcial de las encuestas recibidas, que se informó a los participantes. Se ofreció a los médicos del país participantes en la investigación ser mencionados en la publicación de los resultados como médicos colaboradores y si alguno colaborara con más de 10 casos de FPI/NIU se le anunció que participarían como coautor. Se envió el protocolo al Comité de Ética del Servicio de Salud Metropolitano Oriente que aprobó su realización (Acta del 3.03.2015).

\section{Resultados}

Se invitó a participar a 200 especialistas de acuerdo al registro de la Sociedad Chilena de Enfermedades Respiratorias y respondieron a la invitación 77 (38,5\%). Sin embargo, participaron enviando casos 40 médicos (20\%); 17 de los cuales enviaron 10 o más casos en el período de
1 año. Los casos fueron 700 y provinieron de 13 regiones del país (Tabla 1 ).

Los casos se registraron como casos nuevos o antiguos según se trataba de controles o casos nuevos en la consulta del médico. De los 694 casos con registro del antecedente, (en 6 no se registró) fueron nuevos 239 (34\%) y antiguos $455(65,56 \%)$. De los casos nuevos el 80\% tenían menos de 36 meses de historia de enfermedad y en los casos antiguos el $64 \%$ tenían más de 36 meses de historia de enfermedad

La distribución según sexo mostró un 54\% de mujeres y un $46 \%$ de hombres.

En relación a la clasificación la gran mayoría, 509 casos o sea el 73,3\% de los casos fueron clasificados como NIU y 163 o sea el $24 \%$ fueron clasificados como posibles NIU Hubo además 18 casos clasificados como no NIU y hubo 4 casos en blanco. En hombres y mujeres hubo una diferencia en relación a este punto. En hombres el $79,5 \%$ fueron clasificados como NIU y el $20,4 \%$ como posibles NIU. En mujeres el 27,5\% fueron clasificados como posible NIU.

La edad promedio encontrada fue de $71 \pm$ 9,4 años (rango 43 a 97 años) sin diferencia entre hombres y mujeres. Hubo 22 casos $(3,1 \%)$ entre 41 y 50 años, 90 casos entre 51 y 60 años $(12,8 \%) 222$ casos entre 61 y 70 años $(31,7 \%)$ ,256 casos $(36,6 \%)$ entre 71 y 80 años y 109 casos $(15,6 \%)$ sobre 80 años (Figura 1).

Tabla 1. Procedencia de los casos registrados de FPI según Región de consulta, período 2015-2017

\begin{tabular}{|lccc|}
\hline Región & $\begin{array}{c}\text { Casos } \\
\text { (n) }\end{array}$ & $\begin{array}{c}\text { Neumólogos participantes } \\
(\mathbf{n})\end{array}$ & $\begin{array}{c}\text { Habitantes } \\
(\mathbf{n ~ x ~ 1 0})\end{array}$ \\
\hline Tarapacá & 8 & 1 & 324 \\
\hline Antofagasta & 43 & 1 & 599 \\
\hline Atacama & 35 & 1 & 285 \\
Coquimbo & 18 & 1 & 724 \\
\hline Valparaíso & 82 & 5 & 1.790 \\
\hline Región Metropolitana & $330^{*}$ & 21 & 7.036 \\
\hline O'Higgins & 10 & 2 & 908 \\
\hline Maule & 53 & 1 & 1.033 \\
Biobío & 60 & 3 & 2.018 \\
\hline Araucanía & 13 & 2 & 938 \\
\hline De los Ríos & 37 & 2 & 380 \\
\hline Los Lagos & 5 & 1 & 823 \\
\hline Aysén & 5 & 1 & 102 \\
\hline Magallanes & 1 & 1 & 165 \\
\hline Total & 700 & 43 & 17.125 \\
\hline
\end{tabular}

*Se excluyeron 6 casos por no corresponder a FPI. 


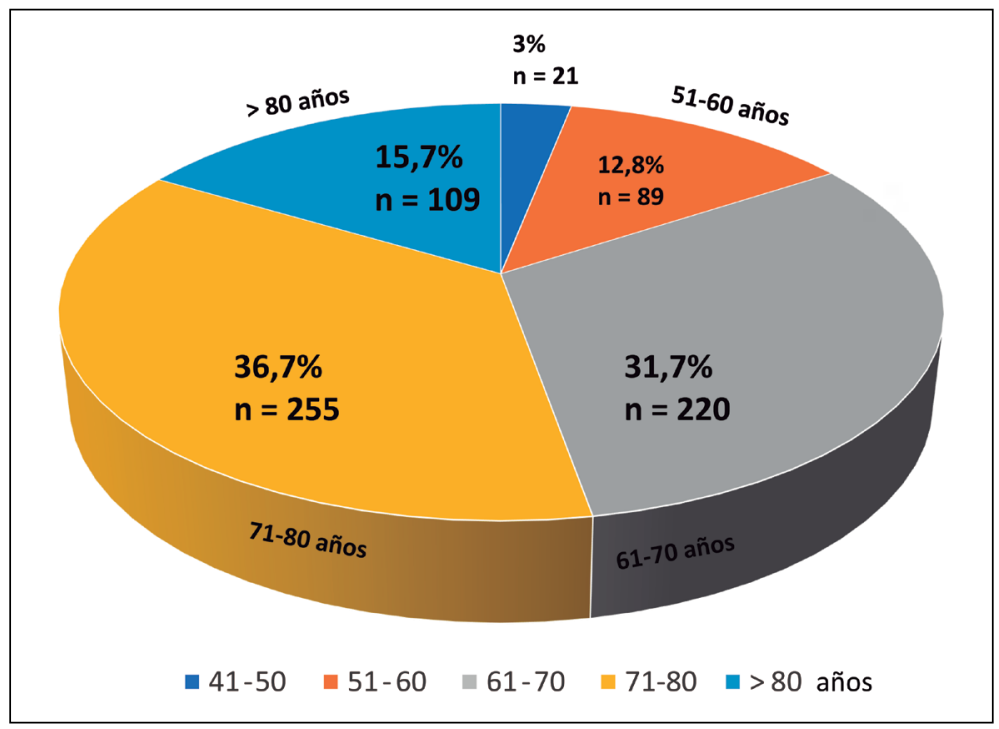

Figura 1. Distribución por edad de 699 casos de Fibrosis Pulmonar Idiopática registrados por neumólogos en Chile entre junio de 2015 a junio de 2017.
La encuesta consideró reporte de antecedentes o sintomatología evidente de mesenquimopatía. Hubo 94 pacientes que nos dieron este antecedente lo que constituye el $13 \%$ de los casos siendo el $80 \%$ de sexo femenino.

Se preguntó adicionalmente por exposiciones de diversa naturaleza (drogas, aves y hongos en casa) En 76 casos hubo este antecedente, $(10,8 \%)$ principalmente de exposición a aves (Tabla 2).

En 110 casos se realizó biopsia pulmonar correspondiendo al 15,8\% de todos los casos. De éstos, en 18 casos era biopsias transbronquiales $(16,3 \%)$ y en 92 la biopsia se obtuvo a través de videotoracoscopía $(83,6 \%)$.

\section{Discusión}

En nuestro conocimiento este representa el primer estudio disponible en la actualidad que intenta obtener algunos preliminares sobre la realidad de la Fibrosis Pulmonar Idiopática en Chile. Ya esbozábamos en la introducción los escasos antecedentes que existen sobre la epidemiología de la enfermedad y las dificultades de realizarlos. Por otro lado esta información debería facilitar una mejor planificación de la atención de esta enfermedad la que pareciera estar aumentando en el mundo. Esta información permite disponer de una línea basal preliminar sobre la condición de los pacientes diagnosticados, considerando que el esquema terapéutico en uso por largo tiempo no resultó eficaz y fue más bien deletéreo. Afortunadamente y en forma casi simultánea con el conocimiento de la ineficacia de las terapias
Tabla 2. Características demográficas y clínicas de los pacientes incluidos en el registro

\begin{tabular}{|lcc|}
\hline $\begin{array}{l}\text { Características demográficas y } \\
\text { clínicas }\end{array}$ & n & \% \\
Casos totales & 694 & 100 \\
Hombres & 322 & 46 \\
Casos nuevos & 239 & 34 \\
Antecedentes de mesenquimopatías & 94 & 13 \\
\hline Antecedente de exposiciones* & 76 & 11 \\
NIU & 509 & 72,7 \\
Posible NIU & 164 & 24 \\
\hline Biopsia pulmonar & 108 & 15,7 \\
\hline Edad promedio y rango (años) & 71,9 & $43-97$ \\
\hline *Exposición a: aves, drogas y hongos domiciliarios.
\end{tabular}

en uso han aparecido dos nuevos fármacos que muestran utilidad en el manejo terapéutico de esta condición, fármacos que lamentablemente están disponibles para un número muy reducido de los pacientes del sistema público de salud.

La realización de un estudio de prevalencia e incidencia es caro y complejo y excede los objetivos de esta comunicación. No obstante estos resultados establecen una situación basal de utilidad para dichos estudios. El análisis de indicadores de frecuencia es relevante pues de demostrarse que la incidencia aumenta y la prevalencia disminuye, debiera investigarse un diagnóstico tardío y disminución de la sobrevida; si en cambio la incidencia resultara estable y la prevalencia aumenta (como parece derivarse 
de los antecedentes comentados previamente) dispondremos de antecedentes para reforzar intervenciones terapéuticas efectivas.

La Fibrosis Pulmonar Idiopática plantea problemas de manejo que el médico no especialista no domina y por lo tanto generalmente deriva estos pacientes al especialista, por lo que estos resultan una fuente de información relativamente fidedigna, considerando que muchos especialistas participantes se han formado en el Instituto Nacional del Tórax.

La tasa de participación de $20 \%$ es menor que la respuesta de los médicos franceses que en encuesta dirigida a 2.608 neumólogos franceses respondieron el $48 \%{ }^{14}$ Quienes respondieron en nuestro caso reportaron de casi todas las regiones del país con una distribución de casos ingresados concordante con el marco de población general. De los 40 neumólogos participantes 17 contribuyeron con 10 o más casos y, como se preveía un alto porcentaje de ellos correspondió a pacientes en control habitual constituyendo los casos de reciente ingreso un tercio de la muestra. La gran mayoría de los casos nuevos tenían menos de 36 meses de enfermedad.

De los 40 neumólogos participantes 17 completaron 10 o más casos $\mathrm{y}$, como se había ofrecido pasaron a ser coautores; los demás se mencionan en la red creada de FPI al final de este trabajo.

La distribución etaria de la muestra está de acuerdo con lo conocido de la enfermedad. El $84 \%$ de los casos tenían más de 60 años y el $16 \%$ más de 80 años.

Esto está en concordancia con la observación universal y se dispone de antecedentes en la literatura actual que fundamenta adecuadamente la relación FPI-envejecimiento ${ }^{15}$. Llama la atención la distribución de género que obtuvimos. 54\% de los casos correspondieron a mujeres y $46 \%$ a hombres. Si bien la diferencia es pequeña, discrepa de la mayoría de las publicaciones en las que predominan los pacientes de sexo masculino ${ }^{16-17}$. Es interesante señalar que hay algunas publicaciones de países remotos (Egipto, Pakistán) que comunican series de pacientes FPI con mayoría de mujeres ${ }^{18-19}$. No conocemos la causa de esta diferencia aunque pudiera ser que en nuestra serie hay más mesenquimopatías clínicamente no evidentes.

En relación a la distribución de los casos entre NIU y posible NIU, nuestros hallazgos se acercan a otras investigaciones con un 23\% considerados como posibles NIU ${ }^{23}$. Sin embargo hubo diferencias entre hombres $(20,4 \%)$ y mujeres $(27,5 \%)$ Esto pudiera estar en concordancia con lo sugerido en el párrafo anterior ya que es precisamente en el grupo de posibles NIU donde están las mesenquimopatías con compromiso pulmonar más frecuentes. En esta misma línea están los 90 casos $(13 \%)$ en que la pregunta de antecedentes de mesenquimopatía es respondida afirmativamente y esto ocurrió en el $80 \%$ en el sexo femenino. Hay que considerar que no se investigó autoanticuerpos marcadores de mesenquimopatías lo cual hoy se recomienda muy especialmente en los casos de posible NIU. El antecedente de 10\% de auto-reporte de exposiciones ambientales diversas en su origen es interesante, considerando lo comunicado en la literatura ${ }^{20}$ que reporta un alto porcentaje calificados como FPI/NIU y que realmente correspondieron a neumonitis por hipersensibilidad crónicos.

En cuanto a la a la utilización de biopsia pulmonar en el diagnóstico de la enfermedad en Chile en el $15,8 \%$ de la muestra se utilizó la biopsia y en $13,25 \%$ se realizó videotoracoscopía. Este porcentaje parece menor que lo obtenido en otras series ${ }^{21}$ lo cual puede explicarse por la dificultad de realizar el procedimiento en muchas partes del país. En todo caso como se ha reportado en la literatura la tendencia en los últimos años ha sido el disminuir las biopsias en esta enfermedad lográndose el diagnóstico por otros medios ${ }^{21}$.

Nuestro trabajo tiene limitaciones que tenemos claras. La recogida de datos fue hecha por muchos médicos. No obstante, la disponibilidad de un breve protocolo e instructivo, además de entrenamiento clínico homogéneo y disponibilidad de consultas por el grupo de investigadores, reduce el riesgo de variabilidad de sus reportes.

Dado el objetivo de la encuesta, destinada a obtención de antecedentes generales básicos, pero esenciales en la mejor caracterización de le enfermedad en nuestro medio, no recogió datos sobre la situación clínica ni funcional de los pacientes y los datos sobre antecedentes de mesenquimopatias fueron auto reportados.

En síntesis el trabajo realizado permitió obtener información de un conjunto mínimo de datos informados por especialistas neumólogos tratantes, permitiendo una mejor caracterización de pacientes chilenos con esta condición, facilitando el diseño de estrategias de estudios posteriores que den continuidad a este reporte.

\section{Agradecimientos}

Los autores agradecen al Dr. Francisco Arancibia H. Presidente de la Sociedad Chilena de Enfermedades Respiratorias por su apoyo en la colaboración de la Sociedad en el programa 
computacional. Agradecemos también al Laboratorio Abbott y particularmente a la Srta. Ximena Muñoz que desde el comienzo del proyecto se interesó y colaboró con nosotros. *Nómina de colaboradores que ingresaron pacientes: M. Aguayo C., G. Alvear T., C. Andrade V., C. Azócar B., A. Baeza G., X. Cea B., K. Czischke L., M. De la Prida C., R. Fuentes, R. Gil D., M. Henríquez T, C. Inzunza P., A. Jalilie E., R. Jara D., J. Pavie G., G. Santana C., M. Tapia G., C. Valenzuela D., J. L. Velásquez M., J. Villalobos Z., R. Zambrano Z., S. Zenteno G., R. Zúñiga N.

\section{Bibliografía}

1.- RAGHU G, ANSTROM K, KING T, LASKY J, MARTÍNEZ F. Prednisone, Azathioprine and NAcetylcysteine for Pulmonary Fibrosis. N Engl J Med 2012; 366 (21): 1968-77.

2.- KING T, BRADFORD WZ, CASTRO BERNARDINI S, FAGAN E, GLESPOLE I, GLASSBERG $M$, et al. A Phase 3 Trial of Pirfenidone in patients with Idiopathic Pulmonary Fibrosis. N Eng J Med 2014; 370 : 2083-92.

3.- RICHELDI L, DU BOIS R, RAGHU G, AZUMA A, BROWN K, COSTABEL U, et al. Efficacy and Safety of Nintedanib in Idiopathic Pulmonary Fibrosis. N Eng J Med 2014; 370: 2071-82.

4.- THOMEER M, DEMEDTS M, VANDEURZEN K. Registration of interstitial lung diseases by 20 centers of Respiratory Medicine in Flanders. Acta Clin Belg 2001; 56: 163-72.

5.- FELL CH D, MARTÍNEZ, LIU LX, MURRAY S, HAN MK, KAZEROONI EA, et al. Clinical predictors of a diagnosis of idiopathic Pulmonary Fibrosis. Am J Respir Crit Care Med 2010; 181: 832-7.

6.- DEMEDTS M, WELLS A, ANTO JM, COSTABEL U, HUBBARD R, CULLINAN P, et al. Interstitial Lung Diseases: an epidemiological overview. Eur Respir J 2001; 18: Suppl. 32: 2s-16s.

7.- THOMEER JM, COSTABEL U, RIZATO G, POLETTI V, DEMETS M. Comparison of registries of interstitial lung diseases in three European Countries. Eur Respir J 2001; 18: Suppl. 32: 114s-8s.

8.- The International Clsssification of Diseases, 10th revision. Clinical Modification. Disponible en: http;//www. cdc.gov/nchs/icd/icd/0cm.htm.

9.- RAGHU G, CHEN SY, YEH WS, MARONI B, LI Q, LEE YC, COLLARD HR. Idiopathic pulmonary fibrosis in US Medicare beneficiaries aged 65 years and older: incidence, prevalence and survival, 2001-11. Lancet Respir. Med 2014; 2: 566-72.

10.- GRIBBIN J, HUBBARD R, LE JEUNE I, SMITH CJ, WEST CJ, TATA LJ. Incidence and mortality of idiopathic pulmonary fibrosis and sarcoidosis in the UK.
Thorax 2006; 61: 980-5.

11.- NAVARATNAM V, FLEMING KM, WEST J, SMITH CJ, JENKINS RG, FOGARTY A, HUBBARD RB. The rising incidence of idiopathic pulmonary fibrosis in the U.K. Thorax 2011; 66: 462-7.

12.- NATSUIZAKA M, CHIBA H, KURONUMA K, OTSUKA M, KUDO K, MORI M, et al. Epidemiologic survey of Japanese patients with idiopathic pulmonary fibrosis and investigation of ethnic differences. Am. J. Respir. Crit. Care Med 2014; 190: 773-9.

13.- RAGHU G, COLLARD H, EGAN J, MARTINEZ F, BEHR J, BROWN H, et al. An official ATS/ERS/JRS/ ALAT statement-idiopathic pulmonary fibrosis: evidence based guidelines for diagnosis and management. Am J Respir and Crit Care Med 2011; 183: 788-824.

14.- COTTIN, CADRANEL J, CRESTANI B, DOLPHIN J, DELEVAL D, ISRAEL-BIET D, et al. Management of Idiopathic Pulmonary Fibrosis in France: a survey of 1244 pulmonologist. Resp Medicine 2014; 108 (1): 195-202.

15.- SELMAN M, BUENDÍA-ROLDÁN I, PARDO A. Ageing and Pulmonary Fibrosis. Rev. Invest. Clin 2016; 68 (2): $75-83$.

16.- HAN MK, MURRAY S, FELL CD, FLAHERTY KR, TOEWS GB, MYERS J, et al. Sex differences in physiological progression of Idiopathic Pulmonary Fibrosis. Eur Respir J 2008; 31 (6): 1183-8.

17.- LEY B, COLLARD H. Epidemiology of Idiopathic Pulmonary Fibrosis. Clinical Epidemiology 2013; 5: 483-92.

18.- RASHAD MA, AHMEL K, IBRAHIN A. Idiopathic Pulmonary Fibrosis in Upper Egypt, a single Center study. Egyptian Journal of Chest Diseases and Tuberculosis 2015; 64 (4): 915-9.

19.- RASUL S, KHALID M, IMRAN N, KAHN S, YOUNUS M. Gender Differences in Clinical Presentation of Idiopathic Pulmonary Fibrosis at Lahore, Pakistan Pakistan Annals 2010; 16 (4): 286-9.

20.- MORELL F, VILLAR AF, MONTERO MA, MUNOZ X, COLBY T. Chronic hypersensitivity pneumonitis in patient diagnosed idiopathic pulmonary fibrosis: a prospective case-cohort study The Lancet Respir Med 2013; 1: 6851-93.

21.- KAARTEENAHO R. The current position of surgical lung biopsy in the diagnosis of idiopathic pulmonary fibrosis Respiratory Research 2013; 14: 43 doi: 10.1186/1465-9921-14-43.

Correspondencia a:

Dr. Álvaro Undurraga Pereira

Instituto Nacional del Tórax

Avda. José M. Infante 717

Santiago,Chile.

Email:alvaroundurragap@yahoo.com 